\title{
Micromagnetic simulations of magnetoelastic spin wave excitation in scaled magnetic waveguides
}

Rutger Duflou, Florin Ciubotaru, Adrien Vaysset, Marc Heyns, Bart Sorée, luliana P. Radu, and Christoph Adelmann

Citation: Appl. Phys. Lett. 111, 192411 (2017);

View online: https://doi.org/10.1063/1.5001077

View Table of Contents: http://aip.scitation.org/toc/apl/111/19

Published by the American Institute of Physics

\section{Articles you may be interested in}

Ultra-low damping in lift-off structured yttrium iron garnet thin films

Applied Physics Letters 111, 192404 (2017); 10.1063/1.5002004

Skyrmion-based multi-channel racetrack

Applied Physics Letters 111, 192413 (2017); 10.1063/1.4994093

Collective spin waves in arrays of permalloy nanowires with single-side periodically modulated width

Applied Physics Letters 111, 192403 (2017); 10.1063/1.5006294

Observation of magnons in $\mathrm{Mn}_{2} \mathrm{Au}$ films by inelastic Brillouin and Raman light scattering

Applied Physics Letters 111, 192409 (2017); 10.1063/1.5001705

Excitation of coherent propagating spin waves in ultrathin CoFeB film by voltage-controlled magnetic anisotropy Applied Physics Letters 111, 052404 (2017); 10.1063/1.4990724

Ultra-low voltage control of magnetic properties in amorphous $\mathrm{MgO}$

Applied Physics Letters 111, 192402 (2017); 10.1063/1.5000129

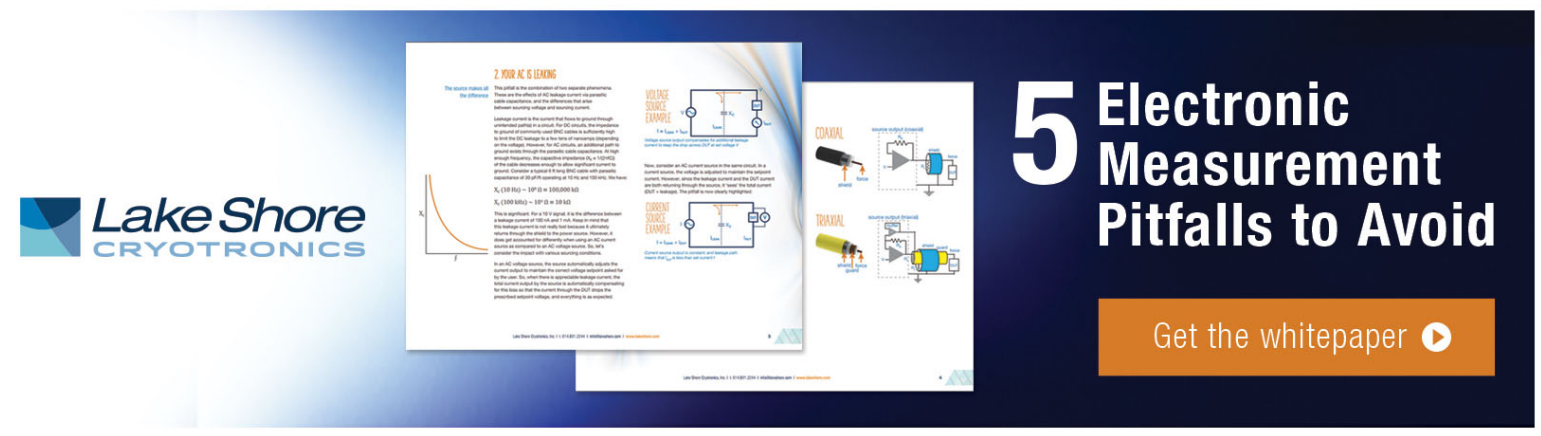




\title{
Micromagnetic simulations of magnetoelastic spin wave excitation in scaled magnetic waveguides
}

\author{
Rutger Duflou, ${ }^{1,2}$ Florin Ciubotaru, ${ }^{1, a)}$ Adrien Vaysset, ${ }^{1}$ Marc Heyns, ${ }^{1,2}$ Bart Sorée, ${ }^{1,2,3}$ \\ luliana P. Radu, ${ }^{1}$ and Christoph Adelmann ${ }^{1, b)}$ \\ ${ }^{1}$ Imec, B-3001 Leuven, Belgium \\ ${ }^{2}$ KU Leuven, Faculteit Ingenieurswetenschappen, B-3001 Leuven, Belgium \\ ${ }^{3}$ Universiteit Antwerpen, Departement Fysica, B-2000 Antwerpen, Belgium
}

(Received 21 August 2017; accepted 20 October 2017; published online 10 November 2017)

\begin{abstract}
We study the excitation of spin waves in scaled magnetic waveguides using the magnetoelastic effect. In uniformly magnetized systems, normal strains parallel or perpendicular to the magnetization direction do not lead to spin wave excitation since the magnetoelastic torque is zero. Using micromagnetic simulations, we show that the nonuniformity of the magnetization in submicron waveguides due to the effect of the demagnetizing field leads to the excitation of spin waves for oscillating normal strains both parallel and perpendicular to the magnetization. The excitation by biaxial normal in-plane strain was found to be much more efficient than that by uniaxial normal out-of-plane strain. For narrow waveguides with a width of $200 \mathrm{~nm}$, the excitation efficiency of biaxial normal in-plane strain was comparable to that of shear strain. Published by AIP Publishing. https://doi.org/10.1063/1.5001077
\end{abstract}

The control and manipulation of ferromagnetic nanostructures using the magnetoelectric effect have recently received increasing attention. ${ }^{1-5}$ Of special interest has been the magnetoelectric generation of spin waves due to a potentially much higher energy efficiency than current-based excitation schemes, e.g., based on inductive coupling to microwaves or on spin torque oscillators. Such magnetoelectric spin wave transducers can therefore be considered as key elements of future low-power magnonic devices. ${ }^{6-10}$

Magnetoelectric excitation and control of ferromagnetic resonance (FMR) as well as propagating spin waves have been studied in both multiferroic materials, such as $\mathrm{BiFeO}_{3},{ }^{11}$ and magnetoelectric compounds. ${ }^{12-18}$ Magnetoelectric compounds consist of piezoelectric and magnetostrictive layers coupled via strain. The strain generated by the piezoelectric layer upon application of an electric field leads to an effective magnetic anisotropy field in the magnetostrictive layer. The resulting magnetization dynamics can then be described by the Landau-Lifshitz-Gilbert equation. While the physics of magnetoelasticity has been established decades ago, ${ }^{19,20}$ the magnetization dynamics in scaled micromagnetic systems have only recently received attention ${ }^{21}$ due to their potential for nanoscale magnonic devices. In this paper, we study the generation of spin waves in scaled ferromagnetic waveguides by a local magnetoelastic transducer for different strain geometries. We show that the demagnetizing fields play a key role and can be used to design efficient magnetoelastic (and thus magnetoelectric) spin wave transducers.

The magnetoelastic energy per unit volume as a function of magnetization $\boldsymbol{m}=\boldsymbol{M} / M_{S}$ (with $M_{S}$ being the saturation magnetization of the ferromagnet) and strain tensor $\boldsymbol{\varepsilon}$ is given in a first-order approximation by ${ }^{22}$

\footnotetext{
${ }^{\text {a)} E l e c t r o n i c ~ m a i l: ~ F l o r i n . C i u b o t a r u @ i m e c . b e ~}$

b)Electronic mail: Christoph.Adelmann@imec.be
}

$$
\begin{aligned}
E_{\mathrm{mel}}= & B_{1}\left[\varepsilon_{x x}\left(m_{x}^{2}-1 / 3\right)+\varepsilon_{y y}\left(m_{y}^{2}-1 / 3\right)+\varepsilon_{z z}\left(m_{z}^{2}-1 / 3\right)\right] \\
& +B_{2}\left(\varepsilon_{x y} m_{x} m_{y}+\varepsilon_{y z} m_{y} m_{z}+\varepsilon_{x z} m_{x} m_{z}\right)
\end{aligned}
$$

Here, $B_{1}$ and $B_{2}$ denote the magnetoelastic coupling constants. The corresponding effective magnetic field, $\boldsymbol{H}$ $=-\nabla_{M} E_{\mathrm{mel}} / \mu_{0}$, is then given by

$$
\boldsymbol{H}=-\frac{1}{\mu_{0} M_{S}}\left(\begin{array}{c}
2 B_{1} \varepsilon_{x x} m_{x}+B_{2}\left(\varepsilon_{x y} m_{y}+\varepsilon_{x z} m_{z}\right) \\
2 B_{1} \varepsilon_{y y} m_{y}+B_{2}\left(\varepsilon_{x y} m_{x}+\varepsilon_{y z} m_{z}\right) \\
2 B_{1} \varepsilon_{z z} m_{z}+B_{2}\left(\varepsilon_{x z} m_{x}+\varepsilon_{y z} m_{y}\right)
\end{array}\right),
$$

with $\mu_{0}$ being the vacuum permeability. It is easy to see that for normal strains parallel or perpendicular to the magnetization, the resulting effective field is parallel to the magnetization or zero, respectively. Therefore, no torque $\tau \propto \boldsymbol{m} \times \boldsymbol{H}$ is exerted on the magnetization in such geometries [see Eq. (S1) in the supplementary material].

Several remedies for this issue are possible, such as the application of strain with a shear component, as in the case, e.g., of Rayleigh surface acoustic waves. ${ }^{13,17}$ Alternatively, a slanted magnetization with respect to a normal strain, due to either an oblique external magnetic field or a canted magnetic anisotropy, ${ }^{6}$ can also lead to nonzero torques. Future magnonic devices may however employ narrow waveguides with dimensions in the $100 \mathrm{~nm}$ range. ${ }^{23}$ In such waveguides, when magnetized transversally (along the $y$-direction), the magnetization is nonuniform due to the nonuniformity of the demagnetizing field in this configuration. This is illustrated in Figs. S1(a) and S1(b) in the supplementary material for $10 \mathrm{~nm}$ thick waveguides with widths of $200 \mathrm{~nm}$ and $500 \mathrm{~nm}$, respectively. These - and all following — simulations were performed with the Object Oriented MicroMagnetic Framework (OOMMF) ${ }^{24}$ using a $5 \times 5 \times 5 \mathrm{~nm}^{3}$ mesh. The parameters of the magnetic waveguide material corresponded to permalloy with an exchange coefficient of $A=1.3 \times 10^{-11} \mathrm{~J} / \mathrm{m}$ and a saturation 
magnetization of $M_{S}=8 \times 10^{5} \mathrm{~A} / \mathrm{m} .^{25}$ As in the spin wave excitation studies below, an external transverse magnetic bias field of $50 \mathrm{mT}$ was applied. The simulation results showed both nonzero $x$ and $z$ components near the edges of the waveguides. Nonuniformities were larger for the narrower waveguide, where nonzero $x$ and $z$ components extended into the center of the waveguide.

The magnetoelastic excitation of spin waves in the narrow waveguides was then simulated using the YY_MEL module ${ }^{25}$ within OOMMF. The external transverse magnetic bias field resulted in Damon-Eshbach-like spin wave modes. The damping constant was assumed to be $\alpha=0.005$, with a gradual increase to a value of 0.8 within $1 \mu \mathrm{m}$ of the ends of the $10 \mu \mathrm{m}$ long waveguide to avoid backreflection of the spin waves. The magnetoelastic coupling constants were $B_{1}=B_{2}=8 \times 10^{5} \mathrm{~J} /$ $\mathrm{m}^{3} .^{26}$ External strains were applied in a $200 \times 200 \mathrm{~nm}^{2}$ region in the center of the waveguides with a sinusoidal amplitude modulation with a frequency of $f=8 \mathrm{GHz}$, well above the FMR frequencies of $5.6 \mathrm{GHz}$ and $4.6 \mathrm{GHz}$ for the 500 and $200 \mathrm{~nm}$ wide waveguides, respectively. The strain was considered to be uniform in the excitation region and quasi-static. The model therefore neglects effects of phonon propagation and phonon-magnon interactions in the rest of the waveguide. Such a situation can be experimentally realized in good approximation, e.g., by including a magnetostrictive layer underneath a piezoelectric actuator that is exchange-coupled to an otherwise nonmagnetostrictive waveguide.

In the following, we discuss the magnetoelastic generation of spin waves in three different excitation geometries: (i) uniaxial normal out-of-plane strain, (ii) biaxial normal in-plane strain, and (iii) in-plane shear strain. We first address the effect of oscillating uniaxial out-of-plane strain (Fig. 1) with all components of the strain tensor being zero except $\varepsilon_{z z}$. Experimentally, this may be realized by a piezoelectric actuator with a top contact exerting stress on the waveguide underneath [Fig. 1(a)]. In this geometry, the generated effective anisotropy field is proportional to $m_{z}=M_{z} / M_{S}$ [see Eq. (2)].

Figures 1(b) and 1(c) show the resulting magnetization oscillation pattern ( $m_{z}$ component) in $200 \mathrm{~nm}$ and $500 \mathrm{~nm}$ wide waveguides, respectively, after excitation for $9 \mathrm{~ns}$ with a uniaxial out-of-plane strain oscillating at $8 \mathrm{GHz}$. All patterns here and below were obtained in the linear regime, i.e., spin wave amplitudes were proportional to the magnitude of the applied strain and the magnetoelastic constants. Detailed quantitative descriptions of the strain tensors used in the simulations can be found in the supplementary material.

The $500 \mathrm{~nm}$ waveguide showed a complex magnetization pattern due to the superposition of multiple spin wave modes. By contrast, the $200 \mathrm{~nm}$ wide waveguide showed a much more uniform wave front. Simulations were also performed with an oscillating external magnetic field, mimicking excitations by the Oersted field of a microwave antenna. In this case, similar mode patterns were observed (data not shown), suggesting that they are inherent to the waveguide rather than to the excitation mechanism. The apparent phase difference at the edges of the $200 \mathrm{~nm}$ wide waveguide can be ascribed to the asymmetry of the magnetization with $m_{z}$ components of opposite signs at the edges. This leads to a phase difference of $\pi$ in the precession of the spins at both edges and the "canting" of the mode.

In both waveguides, we thus observe spin wave excitation by the magnetoelastic effect due to uniaxial out-of-plane strain. As shown in Figs. S1(a) and S1(b), the magnetization was not uniform due to the nonuniformity of the demagnetizing field. This led to nonzero $m_{z}$ in the waveguide. However, due to the shape anisotropy of the film, the $m_{z}$ component was rather small. Therefore, the generated magnetoelastic torques were weak and spin wave amplitudes rather low, as shown in Fig. 1(d), for both $200 \mathrm{~nm}$ and $500 \mathrm{~nm}$ wide

(a)
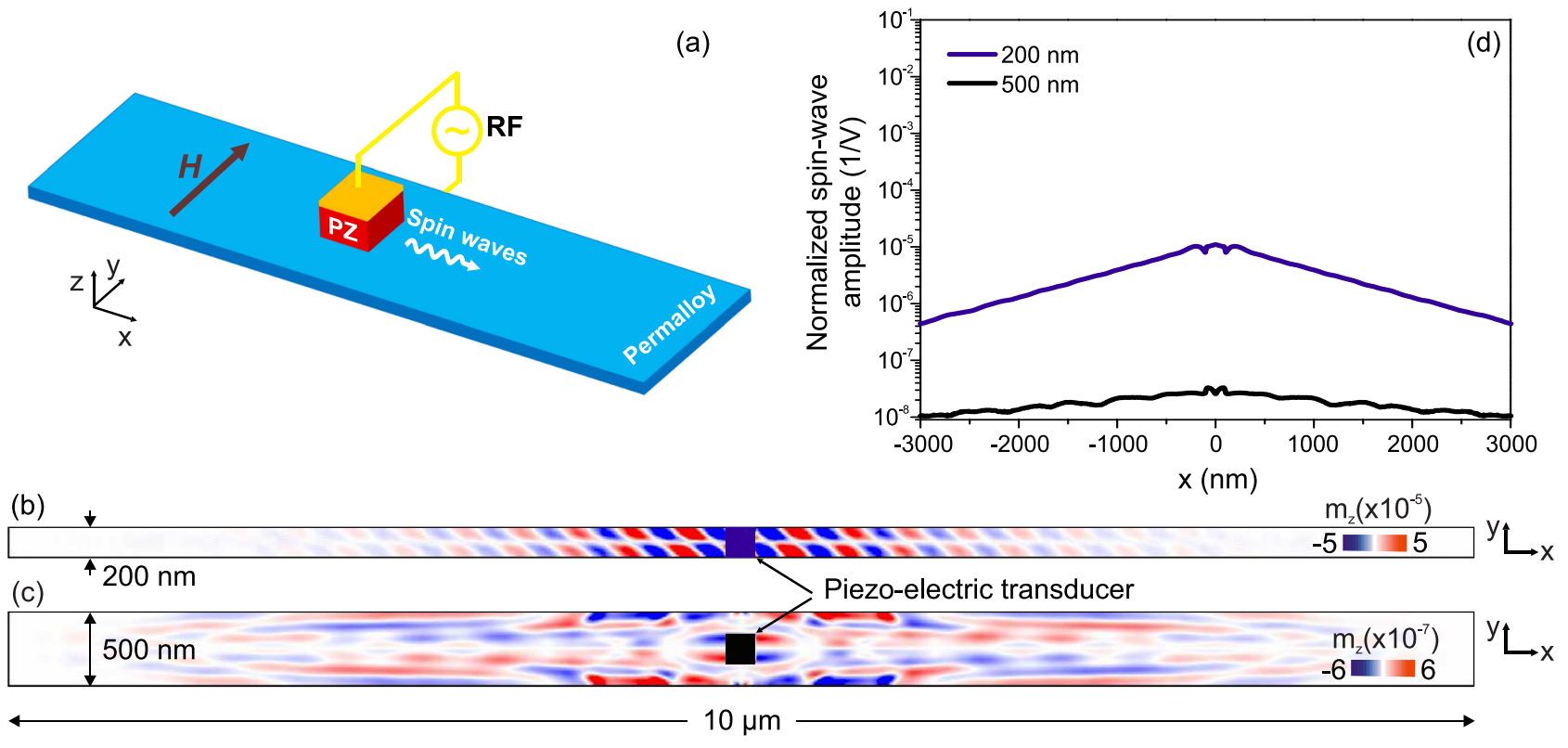

FIG. 1. Uniaxial normal out-of-plane strain: (a) Device design consisting of a piezoelectric pillar (red) on top of the magnetic waveguide (blue). The piezoelectric pillar is actuated by an rf voltage source via a top electrode. $m_{z}$ snapshot images of the magnetization oscillation patterns in (b) $200 \mathrm{~nm}$ and (c) $500 \mathrm{~nm}$ wide waveguides, respectively, after $9 \mathrm{~ns}$ of excitation by uniaxial out-of-plane strain oscillating at $8 \mathrm{GHz}$. The static background magnetization was subtracted to enhance the signal. The applied voltage was $10 \mathrm{~V}$. (d) Corresponding amplitudes (normalized to the excitation voltage and $M_{S}$ ) of the generated spin waves as a function of propagation distance $x$ for both the $200 \mathrm{~nm}$ and $500 \mathrm{~nm}$ waveguides. 
waveguides, respectively. In these graphs, the spin wave amplitude was calculated as the average deviation from the equilibrium magnetization over the cross-section of the waveguide and over one excitation period and normalized to the excitation voltage. The spin wave amplitude was found to be much larger in the $200 \mathrm{~nm}$ wide waveguide than in the $500 \mathrm{~nm}$ wide one, where it was essentially negligible. This can be understood by a larger nonuniformity of the demagnetizing field in the narrower waveguide, resulting in increased $m_{z}$. In all cases, an exponential decay of the spin wave amplitude along the waveguide was observed. According to the analytical calculations of the dispersion relations in uniformly magnetized waveguides, the spin waves at $8 \mathrm{GHz}$ have a higher group velocity in $500 \mathrm{~nm}$ wide waveguides when compared to the $200 \mathrm{~nm}$ case. As the decay length is given by the product of the relaxation time $\tau=1$ / $(2 \pi \alpha f)$ and the group velocity, this qualitatively explains the difference in the decay length observed in Fig. 1(d).

By contrast, the $m_{x}$ component along the waveguide induced by the effect of the demagnetizing field was much larger than $m_{z}$ due to the shape anisotropy of the waveguide. As shown in Eq. (2), this can be exploited by applying $\varepsilon_{x x}$, which leads to a component of the effective anisotropy field proportional to $m_{x}$. This situation can be realized by a normal biaxial in-plane strain, e.g., experimentally by a piezoelectric actuator with side contacts [Fig. 2(a)]. In this case, the strain tensor contains nonzero $\varepsilon_{x x}$ and $\varepsilon_{y y}$ components with opposite signs.

Figures 2(b) and 2(c) display the distribution of the $m_{z}$ component of the magnetization in the two waveguides of $200 \mathrm{~nm}$ and $500 \mathrm{~nm}$ width, respectively, after excitation for $9 \mathrm{~ns}$ by oscillating biaxial in-plane strain. The frequency was $8 \mathrm{GHz}$, as above. In the $200 \mathrm{~nm}$ wide waveguide, the same spin wave mode as in Fig. 1(b) was observed, albeit with a much larger amplitude. Moreover, a much clearer mode pattern became visible in the $500 \mathrm{~nm}$ wide waveguide [Fig. 2(c)]. In the $500 \mathrm{~nm}$ wide waveguide, the magnetization is rather uniform in the excitation area, and thus, the spin wave dynamics are small perturbations of the waveguide at its edges. During propagation, the excited spin waves will radiate towards the center of the waveguide and interference effects might lead to more complex mode patterns observed in this case.

Figure 1(d) shows that the amplitude of spin waves excited by biaxial in-plane strain was about $10^{4}$ times larger than that for uniaxial out-of-plane strain. As discussed above, this can be attributed to a much larger $m_{x}$ component in the waveguide with respect to $m_{z}$ due to shape anisotropy. In addition, for the narrow waveguide, the demagnetizing field led to a spontaneous symmetry breaking, giving rise to an average $m_{x}$ in the whole sample, rather than only at the edges (see Fig. S1 in the supplementary material), further enhancing the spin wave excitation efficiency.

It is instructive to compare the spin wave excitation efficiency in scaled waveguides using biaxial in-plane strain to strain geometries where the torque is nonzero even for uniform magnetization, e.g., for in-plane shear strain with nonzero $\varepsilon_{x y}$. In this case, a term proportional to $m_{y}$ appears in the effective field [see Eq. (2)]. Such shear strains can be experimentally realized by rotating the piezoelectric actuator used to generate biaxial in-plane strain by $45^{\circ}$ around $z$, as shown in Fig. 3(a). A detailed derivation of the used strain tensor and its explicit form can be found in the supplementary material.

Figures 3(b) and 3(c) show snapshot images of $m_{z}$ for the two waveguides of $200 \mathrm{~nm}$ and $500 \mathrm{~nm}$ width, respectively, after excitation for $9 \mathrm{~ns}$ by in-plane shear strain $\varepsilon_{x y}$ oscillating at $8 \mathrm{GHz}$. Amplitudes of the resulting spin waves propagating along the waveguides are shown in Fig. 3(d).

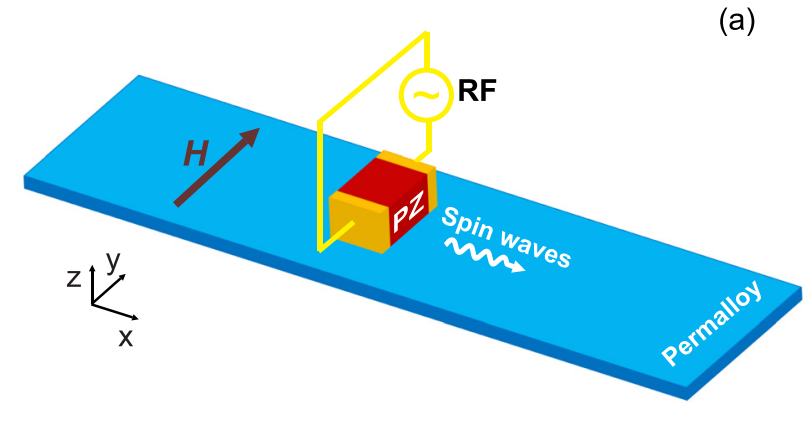

(b)
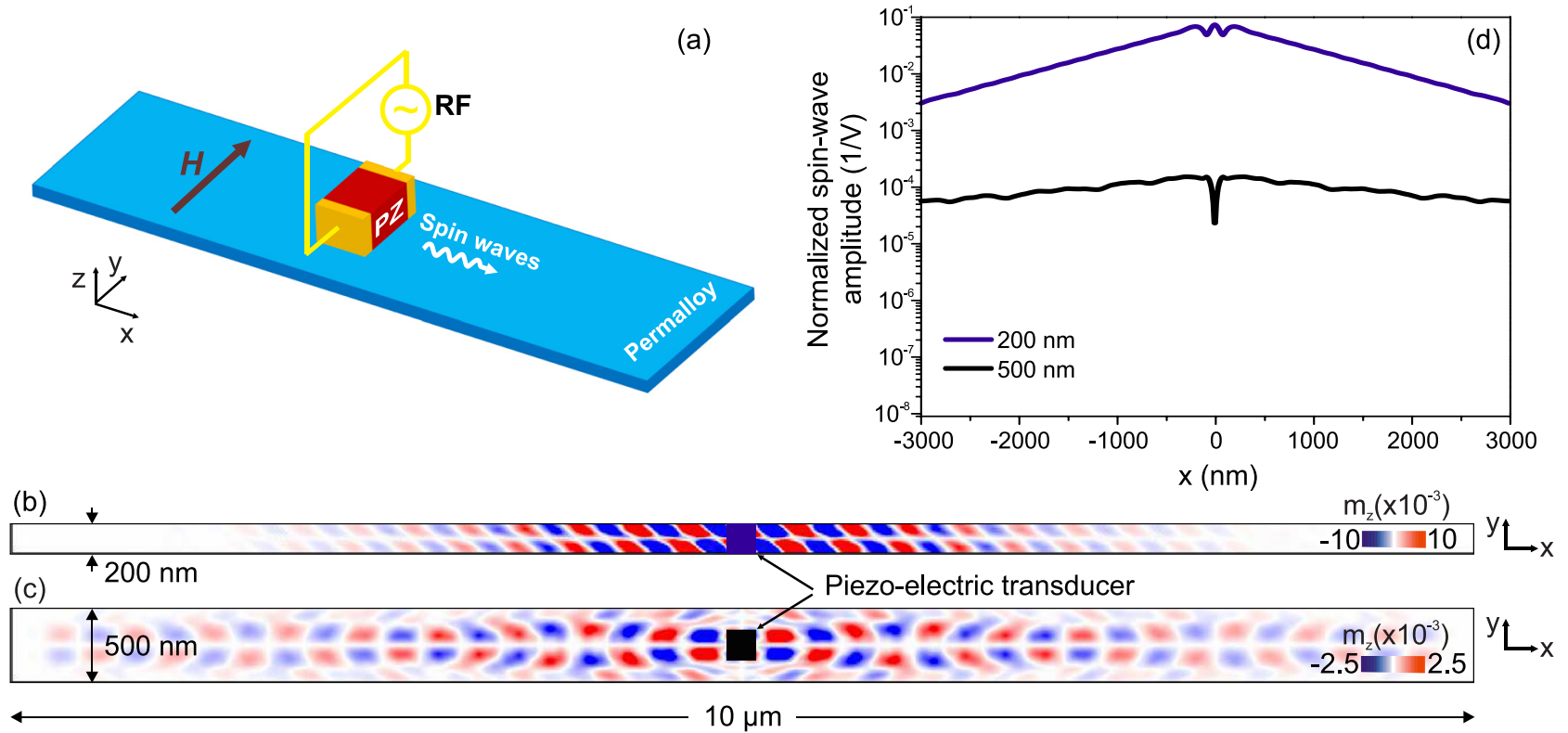

(d) 

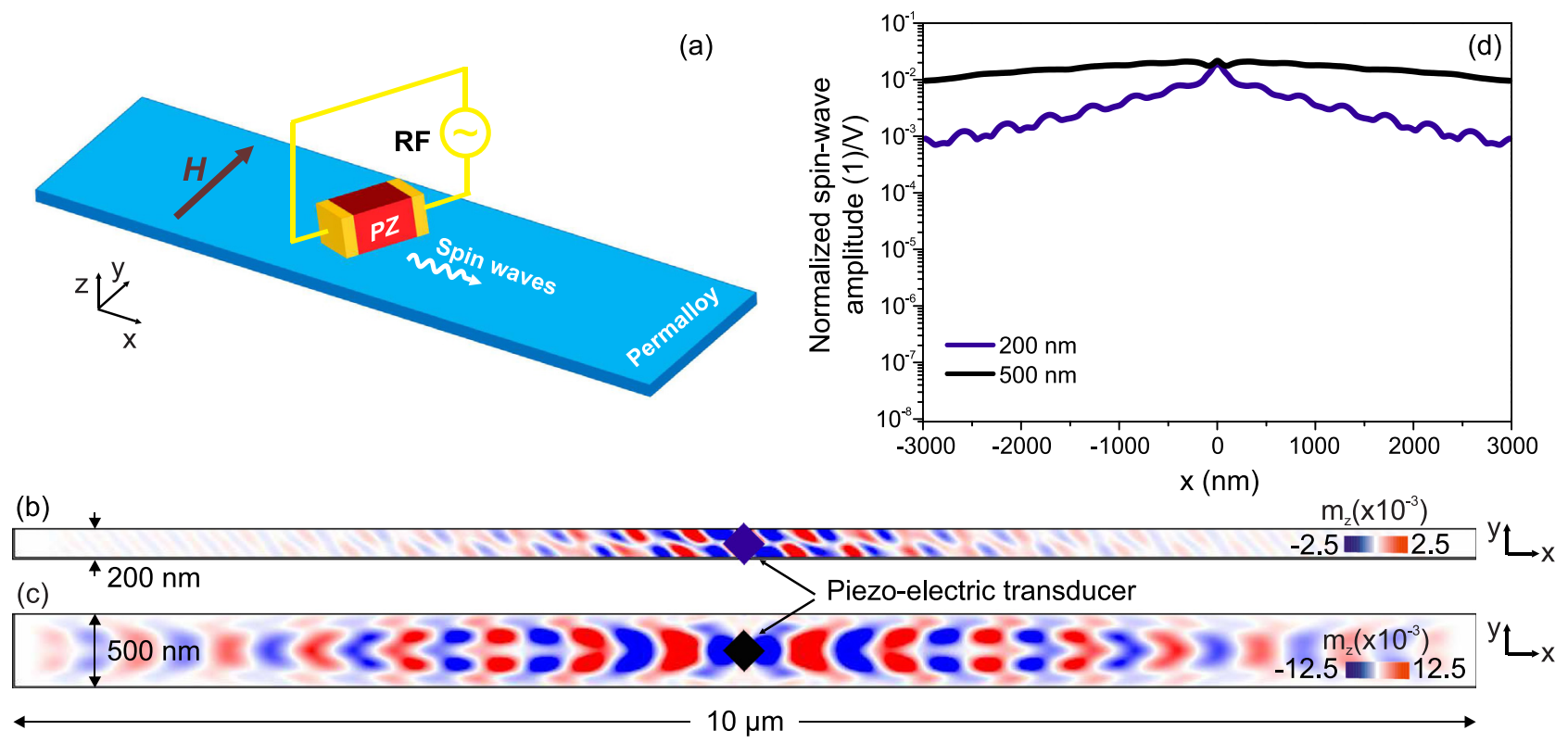

FIG. 3. In-plane shear strain: (a) Device design including a piezoelectric pillar (red) on top of the magnetic waveguide (blue). The pillar is connected to an rf voltage source via two contacts on its sides, and it is rotated by $45^{\circ}$ to generate shear strain. $m_{z}$ snapshot images of the magnetization oscillation patterns in (b) $200 \mathrm{~nm}$ and (c) $500 \mathrm{~nm}$ wide waveguides, respectively, after $9 \mathrm{~ns}$ of excitation by shear strain oscillating at $8 \mathrm{GHz}$. The applied voltage was $1 \mathrm{~V}$. (d) Corresponding amplitudes (normalized to the excitation voltage and $M_{S}$ ) of the generated spin waves as a function of propagation distance $x$ for both the $200 \mathrm{~nm}$ and $500 \mathrm{~nm}$ waveguides.

In contrast to the above cases, the dependence of the spin wave amplitude on the waveguide width was weak since nonuniformities of the demagnetizing field do not play a necessary role in generating torques on the magnetization in this geometry. For a $500 \mathrm{~nm}$ wide waveguide, shear stress led to about two orders of magnitude larger spin wave amplitudes with respect to biaxial in-plane strain. However, for $200 \mathrm{~nm}$ wide waveguides, shear stress was even found to be slightly less efficient in exciting spin waves than biaxial in-plane strain, corroborating the strong impact of the demagnetizing field. While a nonzero $m_{x}$ is strongly beneficial for biaxial in-plane strain and increases the torque on magnetization, it actually decreases the torque in the case of shear strain, as the main torque component is proportional to $m_{y}^{2}-m_{x}^{2}$.

In conclusion, we have studied the excitation of spin waves in scaled magnetic waveguides by the magnetoelastic effect using micromagnetic simulations. In case of a uniform magnetization, normal strains along the principal axes parallel or perpendicular to the magnetization do not lead to torques and therefore cannot excite spin waves. In scaled waveguides, the effects of nonuniform demagnetizing fields lead however to nonzero torques and spin wave generation even for normal strain along principal axes. Biaxial in-plane strain was found to be about four orders of magnitude more efficient than uniaxial out-of-plane strain. In $200 \mathrm{~nm}$ wide waveguides, biaxial in-plane stress was found to be even more efficient than shear stress, which leads to nonzero torques also in uniformly magnetized waveguides. This indicates that magnetoelectric spin wave transducers using biaxial in-plane strain may be highly efficient to excite spin waves in scaled waveguides in a Damon-Eshbach geometry without the need to generate shear strains.

See the supplementary material for an expression of the magnetoelastic torque, detailed quantitative descriptions of the strain tensors used in the simulations, including their derivations, as well as the components of the equilibrium magnetization in 200 and $500 \mathrm{~nm}$ wide waveguides.

${ }^{1}$ M. Fiebig, J. Phys. D: Appl. Phys. 38, R123 (2005).

${ }^{2}$ W. Eerenstein, N. D. Mathur, and J. F. Scott, Nature 442, 759 (2006).

${ }^{3}$ G. Srinivasan, Annu. Rev. Mater. Res. 40, 153 (2010).

${ }^{4}$ C. A. F. Vaz, J. Hoffman, C. H. Ahn, and R. Ramesh, Adv. Mater. 22, 2900 (2010).

${ }^{5}$ S. Fusil, V. Garcia, A. Barthélémy, and M. Bibes, Annu. Rev. Mater. Res. 44, 91 (2014).

${ }^{6}$ A. Khitun and K. L. Wang, J. Appl. Phys. 110, 034306 (2011).

${ }^{7}$ I. P. Radu, O. Zografos, A. Vaysset, F. Ciubotaru, J. Yan, J. Swerts, D. Radisic, B. Briggs, B. Sorée, M. Manfrini, M. Ercken, C. J. Wilson, P. Raghavan, S. Sayan, C. Adelmann, A. Thean, L. Amaru, P. E. Gaillardon, G. D. Micheli, D. E. Nikonov, S. Manipatruni, and I. A. Young, in Proceedings of IEEE International Electron Device Meeting (IEDM) (2015), p. 32.5.1.

${ }^{8}$ S. Dutta, S.-C. Chang, N. Kani, D. E. Nikonov, S. Manipatruni, I. A. Young, and A. Naeemi, Sci. Rep. 5, 9861 (2015).

${ }^{9}$ A. Khitun, D. E. Nikonov, and K. L. Wang, J. Appl. Phys. 106, 123909 (2009).

${ }^{10}$ O. Zografos, M. Manfrini, A. Vaysset, B. Sorée, F. Ciubotaru, C. Adelmann, R. Lauwereins, P. Raghavan, and I. P. Radu, Sci. Rep. 7, 12154 (2017).

${ }^{11}$ P. Rovillain, R. de Sousa, Y. Gallais, A. Sacuto, M. A. Méasson, D. Colson, A. Forget, M. Bibes, A. Barthélémy, and M. Cazayous, Nat. Mater. 9, 975 (2010).

${ }^{12}$ M. Liu, O. Obi, J. Lou, Y. Chen, Z. Cai, S. Stoute, M. Espanol, M. Lew, X. Situ, K. S. Ziemer, V. G. Harris, and N. X. Sun, Adv. Funct. Mater. 19, 1826 (2009).

${ }^{13}$ M. Weiler, L. Dreher, C. Heeg, H. Huebl, R. Gross, M. S. Brandt, and S. T. B. Goennenwein, Phys. Rev. Lett. 106, 117601 (2011); L. Dreher, M. Weiler, M. Pernpeintner, H. Huebl, R. Gross, M. S. Brandt, and S. T. B. Goennenwein, Phys. Rev. B 86, 134415 (2012).

${ }^{14}$ M. Weiler, H. Huebl, F. S. Goerg, F. D. Czeschka, R. Gross, and S. T. B. Goennenwein, Phys. Rev. Lett. 108, 176601 (2012).

${ }^{15}$ M. Liu, B. M. Howe, L. Grazulis, K. Mahalingam, T. Nan, N. X. Sun, and G. J. Brown, Adv. Mater. 25, 4886 (2013).

${ }^{16}$ T. Nan, Z. Zhou, M. Liu, X. Yang, Y. Gao, B. A. Assaf, H. Lin, S. Velu, X. Wang, H. Luo, J. Chen, S. Akhtar, E. Hu, R. Rajiv, K. Krishnan, S. Sreedhar, D. Heiman, B. M. Howe, G. J. Brown, and N. X. Sun, Sci. Rep. 4, 3688 (2014). 
${ }^{17}$ S. Cherepov, P. K. Amiri, J. G. Alzate, K. Wong, M. Lewis, P. Upadhyaya, J. Nath, M. Bao, A. Bur, T. Wu, G. P. Carman, A. Khitun, and K. L. Wang, Appl. Phys. Lett. 104, 082403 (2014).

${ }^{18}$ G. Yu, Z. Wang, M. Abolfath-Beygi, C. He, X. Li, K. L. Wong, P. Nordeen, H. Wu, G. P. Carman, X. Han, I. A. Alhomoudi, P. K. Amiri, and K. L. Wang, Appl. Phys. Lett. 106, 072402 (2015).

${ }^{19}$ C. Kittel, Phys. Rev. 110, 836 (1958).

${ }^{20}$ J. R. Eshbach, J. Appl. Phys. 34, 1298 (1963).

${ }^{21}$ C. Chen, A. Barra, A. Mal, G. P. Carman, and A. Sepulveda, Appl. Phys. Lett. 110, 072401 (2017).

${ }^{22}$ C. Kittel, Rev. Mod. Phys. 21, 541 (1949).
${ }^{23}$ O. Zografos, B. Sorée, A. Vaysset, S. Cosemans, L. Amaru, P. E. Gaillardon, G. De Micheli, R. Lauwereins, S. Sayan, P. Raghavan, I. P. Radu, and A. Thean, in Proceedings of IEEE International Conference on Nanotechnology (IEEE-NANO) (2015), p. 686.

${ }^{24}$ M. J. Donahue and D. G. Porter, OOMMF User's Guide, Version 1.0., Interagency Report NISTIR 6376 (National Institute of Standards and Technology, Gaithersburg, MD, 1999).

${ }^{25}$ Y. Yahagi, B. Harteneck, S. Cabrini, and H. Schmidt, Phys. Rev. B. 90, 140405(R) (2014).

${ }^{26}$ T. Ohtani, T. Kawai, M. Ohtake, and M. Futamoto, EPJ Web Conf. 40, 11004 (2013). 